\title{
Solubility of 5-(2-Chlorophenyl)-7-nitro-1,3-dihydro-1,4-benzodiazepin-2-one, 7-Chloro-1-methyl-5-phenyl-3H-1,4-benzodiazepin-2-one, and 6-(2,3-Dichlorophenyl)-1,2,4-triazine-3,5-diamine in the Mixtures of Poly(ethylene glycol) 600, Ethanol, and Water at a Temperature of $298.2 \mathrm{~K}$
}

\author{
Shahla Soltanpour, ${ }^{\dagger}$ William E. Acree, Jr., ${ }^{*}$ and Abolghasem Jouyban*, ${ }^{\S}$ \\ Biotechnology Research Center, Tabriz University of Medical Sciences, Tabriz 51664, Iran, Department of Chemistry, \\ University of North Texas, Denton, Texas 76203-5070, and Faculty of Pharmacy and Drug Applied Research Center, Tabriz \\ University of Medical Sciences, Tabriz 51664, Iran
}

\begin{abstract}
Experimental solubilities of 5-(2-chlorophenyl)-7-nitro-1,3-dihydro-1,4-benzodiazepin-2-one (clonazepam), 7-chloro-1-methyl-5-phenyl-3H-1,4-benzodiazepin-2-one (diazepam), and 6-(2,3-dichlorophenyl)-1,2,4triazine-3,5-diamine (lamotrigine) in poly(ethylene glycol) (PEG) 600 (1) + ethanol (2), PEG 600 (1) + water (3), and PEG 600 (1) + ethanol (2) + water (3) mixtures at $T=298.2 \mathrm{~K}$ are reported. The Jouyban-Acree model was used to fit the solubility data of each drug in the ternary and sub-binary solvent mixtures in which the mean deviations for clonazepam, diazepam, and lamotrigine were $6.1 \%, 8.3 \%$, and $10.9 \%$, respectively, and the overall value was $8.4 \%$.
\end{abstract}

\section{Introduction}

Poly(ethylene glycol)s (PEGs) are linear or branched neutral polyethers and are available in a variety of molecular weights. The number that denotes the approximate molecular weight of PEG is written immediately following the polymer name. There are several commercially available PEGs with different viscosities and densities with the molecular weight ranging from PEG 200 to PEG 36000. PEG 200 to PEG 800 are in liquid form, whereas PEG 1000 and higher molecular weights are solids. Liquid PEGs are commonly used as cosolvents for the solubilization of drugs in preclinical and clinical studies. ${ }^{1}$ Because of strong H-bonding between PEGs and water, they are freely soluble in water and in many organic solvents. PEGs have variety of applications in the pharmaceutical, chemical, cosmetic, and food industries. ${ }^{2}$ Their low toxicity and high water solubility enable their use for purification of biological materials. They are used in food packaging, as plasticizers, solvents, water-soluble lubricants for rubber molds, wetting or softening agents, or antistatics in the production of urethane rubber and also as components of detergents. In medicinal applications, PEGs are used in cosmetics, ointments, suppositories, ophthalmic solutions, and sustained-release oral pharmaceutical formulations. ${ }^{3}$ In the present study, PEG 600 is used to increase the solubility of 5-(2-chlorophenyl)-7-nitro-1,3-dihydro-1,4-benzodiazepin-2one (clonazepam), 7-chloro-1-methyl-5-phenyl-3H-1,4-benzodiazepin-2-one (diazepam), and 6-(2,3-dichlorophenyl)1,2,4-triazine-3,5-diamine (lamotrigine) in water, in ethanol, and in aqueous ethanol mixtures.

\footnotetext{
* Author to whom correspondence should be addressed. E-mail: ajouyban@ hotmail.com. Fax: +98 4113363231.

Biotechnology Research Center, Tabriz University of Medical Sciences. $\div$ University of North Texas.

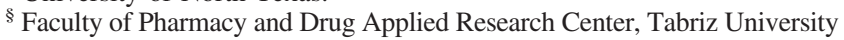
of Medical Sciences.
The solubility of drugs in solvent mixtures has received considerable attention in recent years. Numerous models have been presented for correlation/prediction of the solubility of drugs in mixed solvents. Of the recently reviewed models, ${ }^{4}$ the Jouyban-Acree model is perhaps one of the most versatile models. The model provides very accurate mathematical descriptions for how the solute solubility varies with both temperature and solvent composition. The model for representing the solubility of a solute in binary solvent mixture at various temperatures is ${ }^{4}$

$$
\begin{aligned}
\log C_{\mathrm{m}, T}^{\mathrm{Sat}}=w_{1} \log C_{1, T}^{\mathrm{Sat}}+w_{2} & \log C_{2, T}^{\mathrm{Sat}}+ \\
& {\left[\frac{w_{1} w_{2}}{T} \sum_{i=0}^{2} J_{i}\left(w_{1}-w_{2}\right)^{i}\right] }
\end{aligned}
$$

where $C_{\mathrm{m}, T}^{\mathrm{Sat}}$ is the solute solubility in the solvent mixtures at temperature $T, w_{1}$, and $w_{2}$ are the mass fractions of the solvents 1 and 2 in the absence of the solute, and $C_{1, T}^{\text {Sat }}$ and $C_{2, T}^{\text {Sat }}$ denote the solubility of the solute in the neat solvents 1 and 2 , respectively. The $J$ terms (i.e., $J_{i}, J_{i}^{\prime}$, and $J_{i}^{\prime \prime}$ ) are the constants of the model and are computed by regressing log $C_{\mathrm{m}, T}^{\mathrm{Sat}}-w_{1} \log C_{1, T}^{\mathrm{Sat}}-w_{2} \log C_{2, T}^{\mathrm{Sat}}$ against $\left(w_{1} w_{2}\right) /(T),\left(w_{1} w_{2}\left(w_{1}\right.\right.$ - $\left.\left.w_{2}\right)\right) /(T)$, and $\left(w_{1} w_{2}\left(w_{1}-w_{2}\right)^{2}\right) /(T) .{ }^{4}$ The model for representing the solubility of drugs in ternary solvent mixtures is

$$
\begin{gathered}
\log C_{\mathrm{m}, T}^{\mathrm{Sat}}=w_{1} \log C_{1, T}^{\mathrm{Sat}}+w_{2} \log C_{2, T}^{\mathrm{Sat}}+w_{3} \log C_{3, T}^{\mathrm{Sat}}+ \\
{\left[\frac{w_{1} w_{2}}{T} \sum_{i=0}^{2} J_{i}\left(w_{1}-w_{2}\right)^{i}\right]+\left[\frac{w_{1} w_{3}}{T} \sum_{i=0}^{2} J_{i}^{\prime}\left(w_{1}-w_{3}\right)^{i}\right]+} \\
{\left[\frac{w_{2} w_{3}}{T} \sum_{i=0}^{2} J_{i}^{\prime \prime}\left(w_{2}-w_{3}\right)^{i}\right]+\left[\frac{w_{1} w_{2} w_{3}}{T} \sum_{i=0}^{2} J_{i}^{\prime \prime \prime}\left(w_{1}-w_{2}-w_{3}\right)^{i}\right]}
\end{gathered}
$$

where $C_{3, T}^{\text {Sat }}$ is the solute solubility in the solvent 3 at temperature $T$ and $w_{3}$ is the mass fraction of the solvent 3 in 
Table 1. Details of the Calibration Curves of the Drugs

\begin{tabular}{llcc}
\hline \multicolumn{1}{c}{$\cos$} & \multicolumn{1}{c}{$\begin{array}{c}C \\
\mathrm{~L} \cdot \mathrm{mol}^{-1} \cdot \mathrm{cm}^{-1}\end{array}$} & $\begin{array}{c}c \\
\mathrm{~mol} \cdot \mathrm{L}^{-1}\end{array}$ & $\begin{array}{c}\text { correlation coefficient } \\
(\text { standard error })\end{array}$ \\
\hline clonazepam & 11900 to 12143 & $8.2 \cdot 10^{-6}$ to $8.2 \cdot 10^{-5}$ & $0.999(0.001)$ \\
diazepam & 10835 to 11454 & $2.2 \cdot 10^{-5}$ to $6.7 \cdot 10^{-5}$ & $0.998(0.018)$ \\
lamotrigine & 8283 to 8597 & $2.2 \cdot 10^{-5}$ to $6.6 \cdot 10^{-5}$ & $0.999(0.001)$
\end{tabular}

the absence of the solute. The $J_{i}^{\prime \prime \prime}$ terms are the ternary solvent interaction terms and computed by regressing

$$
\left\{\begin{array}{c}
\log C_{\mathrm{m}, T}^{\mathrm{Sat}}-w_{1} \log C_{1, T}^{\mathrm{Sat}}-w_{2} \log C_{2, T}^{\mathrm{Sat}}- \\
w_{3} \log C_{3, T}^{\mathrm{Sat}}-\left[\frac{w_{1} w_{2}}{T} \sum_{i=0}^{2} J_{i}\left(w_{1}-w_{2}\right)^{i}\right]- \\
{\left[\frac{w_{1} w_{3}}{T} \sum_{i=0}^{2} J_{i}^{\prime}\left(w_{1}-w_{3}\right)^{i}\right]} \\
-\left[\frac{w_{2} w_{3}}{T} \sum_{i=0}^{2} J_{i}^{\prime \prime}\left(w_{2}-w_{3}\right)^{i}\right]
\end{array}\right\}
$$

against $\left(w_{1} w_{2} w_{3}\right) /(T), \quad\left(w_{1} w_{2} w_{3}\left(w_{1}-w_{2}-w_{3}\right)\right) /(T)$, and $\left(w_{1} w_{2} w_{3}\left(w_{1}-w_{2}-w_{3}\right)^{2}\right) /(T)$.

Experimental solubilities of three antiepileptic drugs in ethanol + water mixtures at $T=298 \mathrm{~K}$ were reported in a previous work. ${ }^{5}$ In this work, the experimental solubilities of clonazepam, diazepam, and lamotrigine in PEG $600+$ water, PEG 600 + ethanol, and PEG $600+$ ethanol + water mixtures at $T=298.2 \mathrm{~K}$ are reported. In addition, we illustrate the applicability of the extended form of the Jouyban-Acree model for describing the measured drug solubility data in the ternary and sub-binary solvent mixtures.

\section{Experimental Method}

Materials. Clonazepam and diazepam were purchased from Sobhan Pharmaceutical Company (Iran), and lamotrigine was purchased from Arastoo Pharmaceutical Company (Iran). The purity of the drugs was checked through melting point determinations and a comparison of the measured solubilities in monosolvents with the corresponding data from the literature. ${ }^{5-7}$ Ethanol (with mass fraction purity of 0.995 ) was purchased from Merck (Germany), and PEG 600 was a gift from Daana pharmaceutical company (Iran). Doubledistilled water was used for the preparation of the solutions.

Apparatus and Procedures. The solvent mixtures were prepared by mixing the appropriate amount of the solvents with the accuracy of 0.001 mass fraction. The solubility of clonazepam, diazepam, and lamotrigine in the presence of these two cosolvents was determined by equilibrating an excess amount of drug at $T=298.2 \mathrm{~K}$ using a shaker (Behdad, Tehran, Iran) placed in an incubator equipped with a temperature controlling system maintained constant within $\pm 0.2 \mathrm{~K}$. Because of the high viscosity of PEG 600 , after a sufficient length of time ( $>98 \mathrm{~h}$ ), the saturated solutions of the drugs were centrifuged in $13000 \mathrm{rpm}$ for $0.25 \mathrm{~h}$, diluted with water, and then assayed at $309 \mathrm{~nm}$ for clonazepam, 250 $\mathrm{nm}$ for diazepam, and $306 \mathrm{~nm}$ for lamotrigine, using a ultraviolet-visible (UV-vis) spectrophotometer (Beckman DU-650, Fullerton, U.S.). Concentrations of the diluted solutions were determined from the calibration curves. Details of calibration curves are given in Table 1. Each experimental data point represents the average of at least three repetitive experiments with the measured $\mathrm{mol} \cdot \mathrm{L}^{-1}$ solubilities with $\sigma_{\mathrm{n}-1}$ $=0.00005$ to $\sigma_{\mathrm{n}-1}=0.02788$ being reproducible to within $\pm 3.2 \%$. Densities of the saturated solutions are measured using a $5 \mathrm{~mL}$ pycnometer as a single determination.

Computational Methods. Equation 1 is fitted to the experimental solubility data of each drug in binary mixtures, and the back-calculated solubilities are used to calculate the accuracy of the fit. In the next analysis, eq 2 is fitted to the solubility of drugs in ternary mixtures. The mean relative deviation (MRD) is used to check the accuracy of the prediction methods and is calculated using:

$$
\mathrm{MRD}=\frac{\sum\left\{\frac{\left|\left(C_{\mathrm{m}, T}^{\mathrm{Sat}}\right)_{\mathrm{pred}}-\left(C_{\mathrm{m}, T}^{\mathrm{Sat}}\right)\right|}{\left(C_{\mathrm{m}, T}^{\mathrm{Sat}}\right)}\right\}}{N}
$$

where $N$ is the number of data points in each set.

\section{Results and Discussion}

Tables 2 and 3 list the experimental solubilities of clonazepam, diazepam, and lamotrigine in PEG 600 + water, PEG 600 + ethanol, and PEG 600 + ethanol + water mixtures at $298.2 \mathrm{~K}$ along with the densities of the saturated solutions. There were good agreements among the reported solubilities of clonazepam in water ${ }^{6}\left(0.00012 \mathrm{~mol} \cdot \mathrm{L}^{-1}\right.$ at $T$ $=298.2 \mathrm{~K})$, lamotrigine in water $^{7}\left(0.000664 \mathrm{~mol} \cdot \mathrm{L}^{-1}\right.$ at $T$ $=298.15 \mathrm{~K})$, and diazepam in water ${ }^{8}\left\{0.00014817 \mathrm{~mol} \cdot \mathrm{L}^{-1}\right.$ at temperatures of (295.15 to 297.15 ) $\mathrm{K}$ \} from the literature and the measured solubilities for solubility of clonazepam in water $\left(0.000103 \mathrm{~mol} \cdot \mathrm{L}^{-1}\right.$ at $\left.T=298.15 \mathrm{~K}\right)$, diazepam in water $\left(0.000191 \mathrm{~mol} \cdot \mathrm{L}^{-1}\right.$ at $\left.T=298.2 \mathrm{~K}\right)$, and lamotrigine in water $\left(0.000728 \mathrm{~mol} \cdot \mathrm{L}^{-1}\right.$ at $\left.T=298.2 \mathrm{~K}\right)$, in this work. The minimum solubilities of three drugs are observed in aqueous solutions, and addition of the cosolvents to the aqueous solutions resulted in an increased solubility. The maximum solubility of clonazepam and lamotrigine are observed in neat PEG 600, whereas the maximum solubility of diazepam is achieved in PEG $600+$ ethanol at an ethanol mass fraction of 0.20 .

The Jouyban-Acree model was fitted to the binary and ternary solvent data as described above, and the model constants for clonazepam, diazepam, and lamotrigine solubilities are reported in Table 4. The model provides a reasonably accurate mathematical description of the observed solubility data of the investigated drugs in the three subbinary solvent systems at all cosolvent compositions. This finding is also supported by small MRD values for the backcalculated solubility data. Larger deviations were noted for the ternary PEG $600+$ water + ethanol mixtures. 
Table 2. Experimental Solubilities $C_{\mathrm{m}, T}^{\mathrm{Sat}}$ of Clonazepam, Diazepam, and Lamotrigine in Binary Mixtures of Water (3), Ethanol (2), and PEG 600 (1) at a Temperature of $298.2 \mathrm{~K}$ and Density $\rho$ of the Saturated Solutions

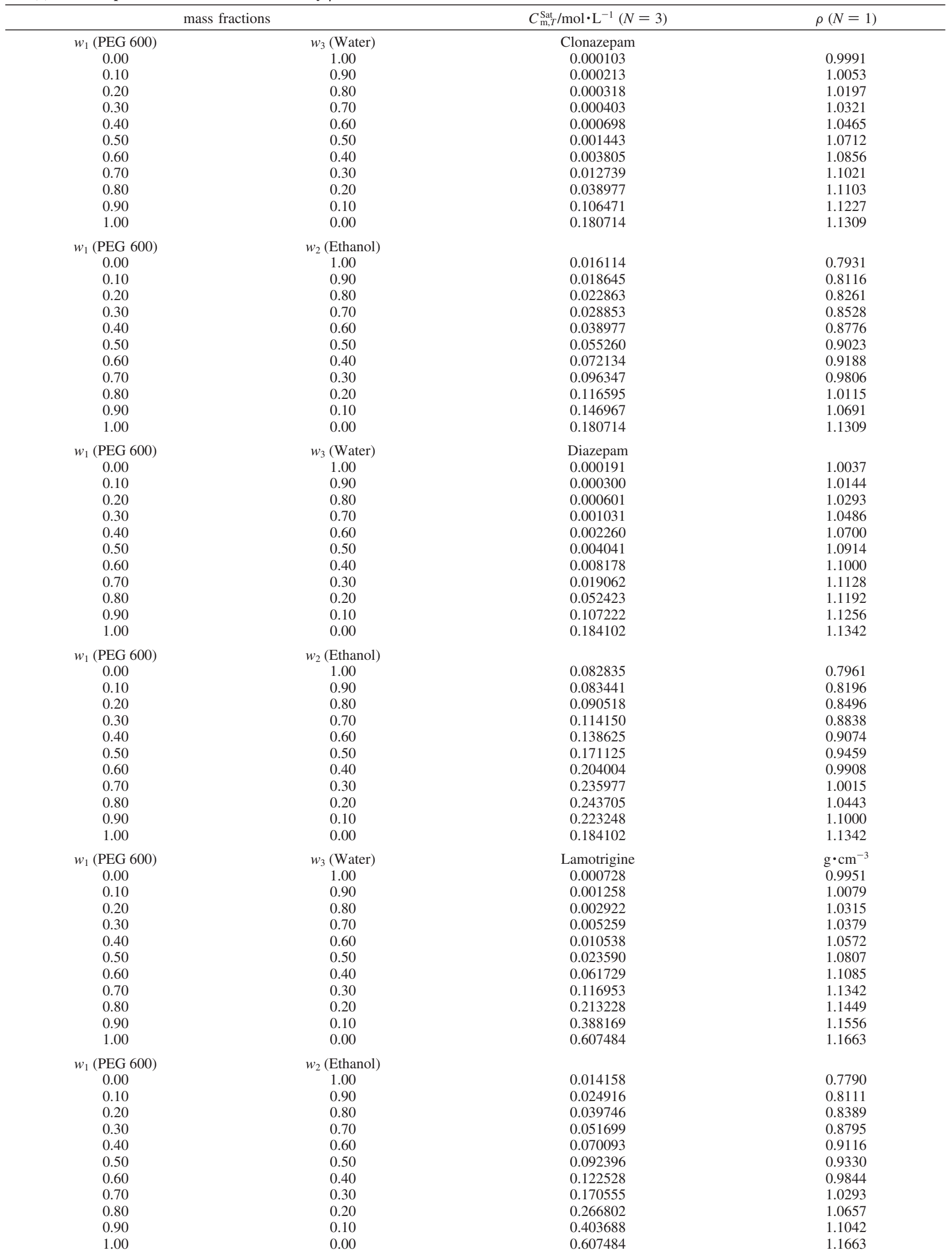


1730 Journal of Chemical \& Engineering Data, Vol. 55, No. 4, 2010

Table 3. Experimental Solubilities $C_{\mathrm{m}, T}^{\text {Sat }}$ of Clonazepam, Diazepam, and Lamotrigine in Ternary Mixtures of PEG $600(1)+$ Ethanol (2) + Water (3) at a Temperature of 298.2 K and Density $\rho$ of the Saturated Solutions

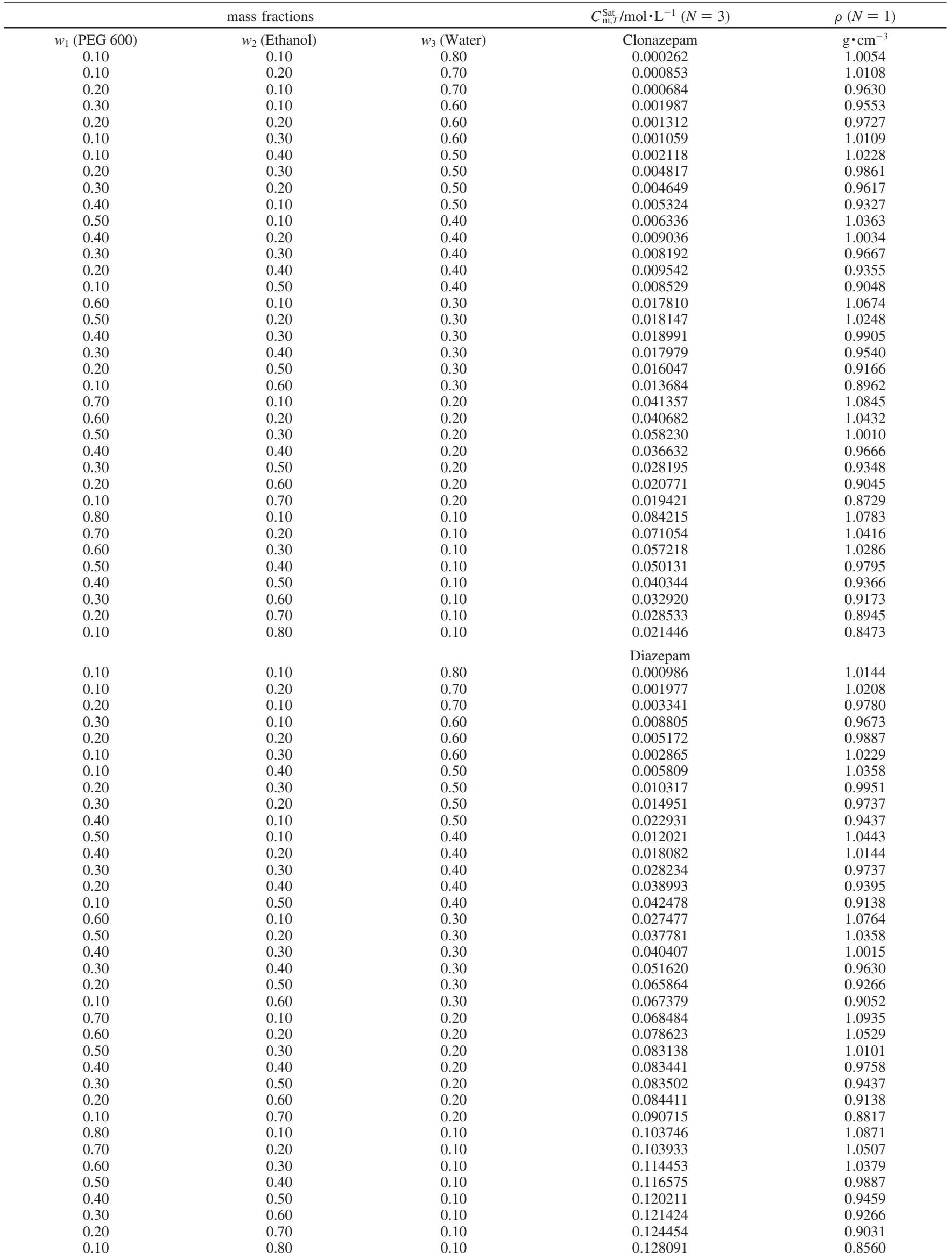


Table 3. Continued

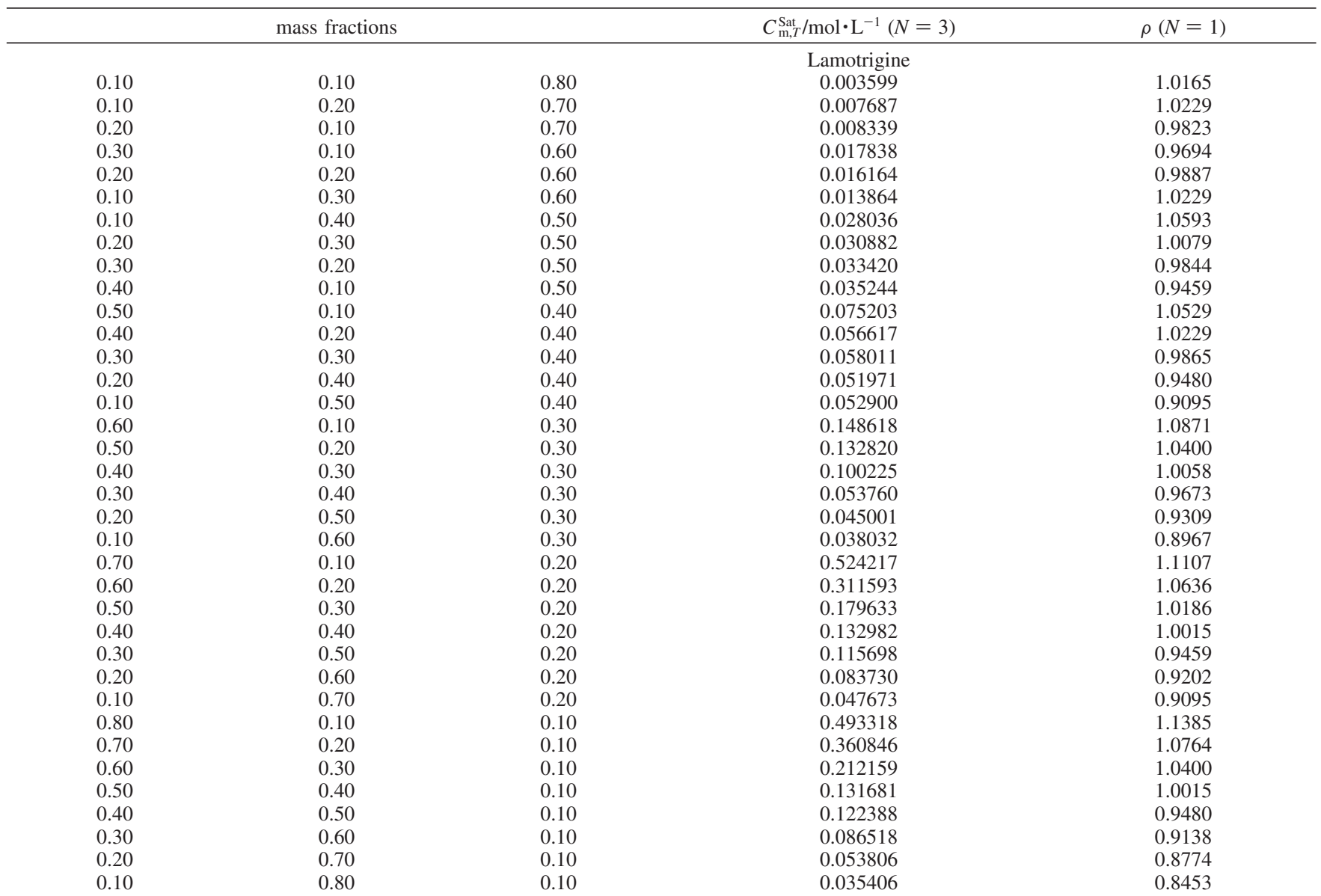

Table 4. Model Constants and the MRDs for the Solubilities of Clonazepam, Diazepam, and Lamotrigine

\begin{tabular}{|c|c|c|c|c|c|}
\hline drug & solvent system & $\begin{array}{c}J_{0} \\
\mathrm{~K}^{-1} \\
\end{array}$ & $\begin{array}{c}J_{1} \\
\mathrm{~K}^{-1} \\
\end{array}$ & $\begin{array}{c}J_{2} \\
\mathrm{~K}^{-1} \\
\end{array}$ & MRD \% \\
\hline \multirow[t]{3}{*}{ clonazepam } & PEG $600(1)+$ ethanol (2) & -13.855 & 146.219 & $a$ & 2.1 \\
\hline & PEG $600(1)+$ water $(3)$ & -564.870 & 280.725 & 1077.110 & 2.0 \\
\hline & PEG $600(1)+$ ethanol $(2)+$ water $(3)$ & 2700.808 & $a$ & $a$ & 13.1 \\
\hline \multirow[t]{3}{*}{ diazepam } & PEG 600 (1) + ethanol (2) & 171.742 & 324.261 & -42.734 & 1.1 \\
\hline & PEG $600(1)+$ water $(3)$ & -195.330 & 192.804 & 283.607 & 6.9 \\
\hline & ethanol $(2)+$ water $(3)^{b}$ & -441.610 & 629.139 & 1474.210 & 5.5 \\
\hline \multirow{3}{*}{ lamotrigine } & PEG $600(1)+$ water $(3)$ & 98.680 & 284.827 & $a$ & 5.9 \\
\hline & ethanol $(2)+$ water $(3)^{b}$ & 53.416 & 813.345 & 2122.283 & 8.0 \\
\hline & PEG $600(1)+$ ethanol (2) + water (3) & 4114.706 & 6521.672 & 14406.844 & 27.7 \\
\hline
\end{tabular}

${ }^{a}$ Not statistically significant with zero. ${ }^{b}$ Experimental data are taken from a previous paper, ${ }^{5}$ and the solvent compositions are converted to mass fractions.

\section{Literature Cited}

(1) Myrdal, P. B.; Yalkowsky, Y. H. Solubilization of Drugs. Encyclopedia of Pharmaceutical Technology; Mercel Dekker Inc.: New York, 1999.

(2) Harris, J. M. Poly(ethylene glycol) Chemistry, Biotechnical and Biomedical Application. Plenum Press: New York, 1992.

(3) Ballantyne, B.; Leung, H. W.; Hermansky, S. J.; Frantz, S. W. Subchronic, Chronic, Pharmacokinetic and Genotoxicity Studies with Polyox Water Soluble Resin. Toxicol. Lett. 1998, 1, 46.

(4) Jouyban, A. Review of the Cosolvency Models for Predicting Solubility of Drugs in Water-Cosolvent Mixtures. J. Pharm. Pharm. Sci. 2008, $11,32-58$.

(5) Shayanfar, A.; Fakhree, M. A. A.; Acree, W. E., Jr.; Jouyban, A. Solubility of Lamotrigine, Diazepam and Clonazepam in Ethanol + Water mixtures at 298.2 K. J. Chem. Eng. Data 2009, 54, 1107-1109.
(6) Neil, M. J.; Smith, A.; Heckelman, P. E. The Merck Index. An Encyclopedia of Chemicals, Drugs and Biologicals, 13th ed.; Merck \& Co Inc.: Rahway, NJ, 2001.

(7) Moffat, A. C. Clarke's Analysis of Drug and Poisons; Pharmaceutical Press: London, 2004.

(8) Loftsson, T.; Hreinsdóittir, D. Determination of Aqueous Solubility by Heating and Equilibration: A Technical Note. AAPS PharmSciTech 2006, 7, e1-e4.

Received for review August 20, 2009. Accepted October 8, 2009. The authors would like to thank Research Affairs, Tabriz University of Medical Sciences, for partial financial support of this work.

JE9005807 